\title{
LAbVIEW : A MODERN DATA ACQUISITION SYSTEM IN AN INTRODUCTORY MECHANICS LABORATORY
}

\author{
Richard A. Young \\ Department of Chemistry and Physics, \\ University of Portland, Portland, OR 97203. \\ young@up.edu
}

\section{Introduction}

As the title suggests, this paper describes our implementation of LabVIEW in our freshman level mechanics laboratory. The laboratory is closely coupled to a traditional lecture based physics course composed of engineering, physical science, mathematics, and computer science students. Over the past decade there has been a significant amount of instructional research and development in the teaching of physics at the introductory level ${ }^{1-10}$. This work has demonstrated that the capability to rapidly acquire and display data provides a valuable tool to promote student experimentation and understanding. As a result a number of computer oriented educational packages that are directed toward the introductory physics laboratory experience have been developed. ${ }^{11-13}$

Rather than adopt one of these packages we decided to employ LabVIEW, a commercially oriented data acquisition environment, in the computerization of our laboratory. This decision was motivated by a number of factors which we wanted our computing environment should provide. Among these were providing:

- Students with an early exposure to a data acquisition environment which is widely used in industry and which could be used in both their upper division courses and post graduation employment.

- The capability to rapidly develop new data acquisition modules so that the laboratory could easily evolve to incorporate new sensors and experiments as needed.

- The opportunity to encourage students to adopt a "systems modeling" approach toward experimental design ${ }^{2}$ through LabVIEW's data flow programming paradigm.

- An easily understood student/computer interface.

The remainder of the paper discusses these points in more detail. Section II provides a brief description of the institutional setting and the laboratory philosophy. Section III describes the hardware and software used in the laboratory, Section IV describes our development activities, Section V illustrates a "typical" experiment using LabVIEW for data acquisition, and finally in Sec. VI we present some concluding remarks.

\section{Institutional and Course Context}


With an enrollment of 2,100, the University of Portland is a private institution whose focus is undergraduate education. The University has an engineering school offering majors in electrical, mechanical, and civil engineering (all ABET accredited). The calculus based introductory physics (mechanics) course is offered once a year with an enrollment of about 110 students, $80 \%$ of which are freshman engineering majors.

The associated laboratory sections, each with 18-26 students, meet on a weekly basis throughout the semester. Many of the lab activities are structured around a two week time frame, and laboratory "hand outs" offer broad guidelines as to what an experiment entails. Students then spend the first week designing the experiment, constructing the apparatus, organizing a data table, taking preliminary data, trying alternative approaches, and doing some "sanity checks" to see if the proposed procedure makes sense. The students' goal for this part of the lab is to write their own "cookbook" which documents the procedure to be followed in the second week of the experiment, when the final data set is taken. Within this context:

- There is a gradual reduction in the amount of direction offered in the lab handouts, coupled with an increased frequency of answering a question with an experimental question.

- There is an active encouragement for creative experimentation (and fun) through a more leisurely and reflective pace of experimental activity. The relaxed pace of the laboratory allows for peer group teaching and learning. Students are free to look at a variety of experimental solutions, generated by their peers, and incorporate a variety of ideas in their own approach.

Prior to computerization, however, alternative experimental approaches often remained unexplored because:

- Data acquisition was often tedious; so the amount of data acquired was limited, and

- Manual plotting of graphs was also tedious and analysis was slow, a frequent question was, "How many data points do we have to take?"

Consequently, the laboratory was hampered in achieving its full potential of encouraging and supporting self-directed, alternative approaches to data acquisition and graphical analysis.

\section{The Computer Supported Laboratory}

To address the need for a modern integrated data acquisition and analysis system the University, with matching support from the NSF-ILI ${ }^{14}$ program, provided $10 \mathrm{PC}$ based data acquisition and analysis systems and a spectrum of probes and sensors for data acquisition.

Our immediate goal was to develop a laboratory computing environment which would:

- retain the spirit and philosophy of independent investigation described above,

- support and develop our students' visual learning style, 
- provide a more flexible environment which would evolve to engage the student in a more reflective activity than making choices from a menu ${ }^{6}$, and

- expose students to a "systems modeling" approach toward experimental design 2 .

Since our physics program does not have the luxury of equipment development and maintenance personnel we had a number of additional practical constraints, including:

- Flexibility to adopt new experiments as opportunities occurred,

- Minimization of software development time to support rapid development of data acquisition programs and to interface with a variety of sensors,

- A common, interface between data acquisition and data analysis to simplify the interaction between these two phases of experimental activity,

- A Graphical User Interface (GUI) for ease of interaction with the computer, and

- Relevance to upper division and industrial laboratories so that students could build a level of expertise that would filter through their education, and into the workplace.

The LabVIEW product from National Instruments together with its $\mathrm{PC}+$ data acquisition card seemed to satisfy all of these criteria and was adopted as our data acquisition/analysis environment.

The current laboratory contains 9 486PC's, running at $33 \mathrm{Mhz}$, and one HP laser printer. These systems are linked via Novell 3.11 ethernet network. Each PC runs Windows 3.1.1 and LabVIEW locally. The network server provides print services, a backup configuration for the PC's, and directories for students to save data and LabVIEW programs. The primary sensors currently used are photo gates, force sensors, and the Sonic Ranger, all from Pasco Scientific.

\section{Development Activities}

\section{A. Software}

As discussed above, the central piece of technology introduced in this laboratory was LabVIEW, a popular data flow programming language used for rapid development of data acquisition systems. At the time the NSF-ILI proposal was funded we were unaware of any other effort to use LabVIEW (or a similar software environment) in the context of an introductory physics laboratory. After funding, we became aware of a similar effort (on a much larger scale and with much larger personnel resources) at Purdue University ${ }^{15}$.

Our approach differs somewhat from Purdue's in that we have attempted to introduce students to elements of data flow programming in the context of conducting their physics experiments. The LabVIEW data flow paradigm encourages the programmer to think about the entire flow of data, from initial acquisition, to preliminary and then final analysis. Since a central element of our laboratory experience is the planning of the experiment, we wanted to leverage LabVIEW's environment to support these planning activities. 
The mastery of the LabVIEW paradigm does, however, require a significant learning curve, and it is unrealistic to have students develop LabVIEW programs from low-level elements while simultaneously trying to understand the implications of $\mathrm{F}=\mathrm{Ma}$. Consequently we developed a number of VI's (LabVIEW elements, and programs are commonly referred to as Virtual Instruments, or VI's) that students could use and that required a limited amount of "wiring" to create a complete data acquisition program. The modules developed were:

i) A VI to acquire data from a photo gate detector. The VI computes the time(s) between the on/off (and off/on) transitions of a photo gate as it is interrupted. If the interruption is due to equally spaced pickets (on a picket fence ) or to equally spaced spokes (on a pulley), then these transition times can be used to obtain a displacement vs. time plot for the object as it passes through the photo gate.

ii) A VI to control and acquire data from a Sonic Ranger. The standard software for the Pasco Sonic Ranger does not run under Windows, or under a DOS window in Windows. To use this sensor with LabVIEW students would then be required to acquire data in the DOS mode, save it to a file, open Windows and LabVIEW, read the data from the file, and then analyze the data. This procedure is awkward and defeats the objective of an integrated acquisition/analysis environment.

A LabVIEW VI which provides high level control of the Sonic Ranger was developed to alleviate this problem.

iii) A preliminary data analyzer VI. After data is acquired it may be passed to this VI which simultaneously shows the position (x), the velocity (v) and the acceleration (a) of the object.

In addition, the analyzer allows the student to expand any region of these curves for closer inspection and then pass the selected region to a second analyzer for extraction of parameters via least squares fitting. Thus the student can analyze any subset of the $(x, v, a)$ data desired. The data can also be recycled so that different segments of the same data set can easily be analyzed individually.

iv) A quantitative data analyzer VI. Data passed to this VI may be plotted on a linear or semi-log plot and fit with a least squares algorithm. The least squares coefficients may be read from the GUI. The data plot is designed to change dynamically, i.e., if a set of data is plotted as y vs. $\mathrm{x}$ and the setting is changed to plot y vs. $\mathrm{x}^{2}$, the plot will reflect the change in real time. This allows students to easily search for data trends and in combination with (iii) provides quantitative support for exploratory data analysis.

v) Utility VI's. Among these are a VI for writing data to a file in a format readable by the standard spreadsheets (Excel and Lotus 123), a VI for smoothing data, and a VI for simulating 
signals from a photo gate when an object is passing through the gate with specified constant acceleration. This latter VI enables students to see the behavior of the photo gate signal and relate this behavior to the displacement vs. time plot.

\section{B. Hardware.}

As discussed above the standard Sonic Ranger software does not operate under the Windows environment, and software control of timing using VI's provides insufficient time resolution when detecting the reflected signals from the Sonic Ranger. Thus, in addition to developing control software, it was also necessary to develop an interface board which times reflection signals by electronic control of counters on the PC+ data acquisition card.

This board was designed to work with the National Instruments PC+ card but a similar design could be used for any data acquisition card that provides access to on card timers. The board, in combination with the Sonic Ranger VI, is capable of detecting reflected pulses from one or two objects over a wide range of distances. In practice the distances are less than 1 meter with a Sonic Ranger pulse interval around $20 \mathrm{msec}$.

\section{Experiments}

To take advantage of the improved data collection environment, several experiments were developed. They are:

\section{Photo gate experiments:}

- An introduction to LabVIEW through analysis of the photo gate signal,

- The elastic properties of a rubber band undergoing collision with an air track glider: A study of the coefficient of restitution as a function of tension in the rubber band,

- Measurement of $g$ using a falling picket fence and Atwood's machine.

Sonic Ranger experiments:

- Work, kinetic, and potential energy in a simple harmonic oscillator,

- Collisions and conservation of energy and momentum,

- Determination of the potential energy function between two repelling magnets,

- Inelastic collisions and damping of vibrations in a modern tennis racket,

- The damped simple harmonic oscillator and design of a magnetic brake.

Force sensor experiments:

- Study of the vibrations of a hack saw blade,

- Force between two repelling magnets.

\section{An example}


The structure of a typical experiment utilizing our LabVIEW VI's may be illustrated by an investigation of the nature of friction torque on a rotating pulley. To study the torque we mounted a Pasco Super Pulley (with ten equally spaced spokes) in the light beam of a photo gate. After manually spinning the pulley we then used the photo gate VI to determine the times when there is a transition in the photo gate light beam. This data was then passed to the $(\mathrm{x}, \mathrm{v}, \mathrm{a})$ VI for display. This VI takes the position and time data as input and numerically differentiates it to also display position, velocity, and acceleration as a function of time. We then selected the velocity data for further quantitative analysis and passed it to the data analyzer VI.

The LabVIEW program which accomplishes all of these steps in sequence is shown in Figure(1) which illustrates the "Front Panel" and the "Block Diagram" for this experiment. These names reflect the general philosophy of data acquisition in LabVIEW. Any data acquisition VI will have a Front Panel on which you can input data to configure the device.

The Front Panel may also have some sort of output indicator, such as a graph or meter scale, which displays the acquired data. In this experiment the necessary output is generated by the $(\mathrm{x}, \mathrm{v}, \mathrm{a})$ and the data analyzer VI's when they are active so no graphs appear on the Front Panel, and we have only the spoke width and acquisition time as control input variables.

Associated with the Front Panel is a wiring diagram that connects the controls to the underlying VI that actually collects the data. In LabVIEW this collection of wires and VI's is called the Block Diagram. When the program is run the execution sequence follows the data flow from left to right, and each VI begins its execution when the necessary data arrives at its input terminals.

Figure(2) shows the (x,v,a) data display as the pulley slows down. Here we can see that taking the second derivative of digitized data, to directly determine the angular acceleration, results in significant amplification of noise. Instead we selected the velocity data and passed it to the data analyzer where two models for the friction torque on the pulley were studied. The first model assumed a constant torque, implying a straight line for the velocity vs. time curve. Figure (3) shows the data and the least squares fit for this model. An alternative model might assume a friction torque proportional to the velocity, and in this case we should obtain a straight line using a semi-log plot of the data. Figure (4) shows the data and least squares fit for this model. Clearly, the constant force model does a better job of describing the friction torque in this case.

\section{Does it work?}

We have not had the luxury of doing a controlled experiment to determine whether our students actually learn and retain more understanding of physics concepts under our computerized environment. We do have some course evaluations and anecdotal evidence that indicates some success and some need for additional work. 
- There is improved student morale regarding the time spent in the laboratory. The lab requires 3 lab hr's/wk for one credit hour, and students are justifiably suspect if they do not perceive an appropriate return on this time investment. Access to the kind of experimental environment that they will encounter in later courses and industry seems to go a long way toward generating relevance to the lab activities.

- When an experiment is misbehaving, and data is not being acquired, students tend to feel out of control. Rather than checking wires, or otherwise altering the experimental arrangement, they often assume it's a software problem and justifiably feel helpless until an instructor arrives.

- There has been a considerable increase in the amount of data taken and in quantitative analysis of that data. Students have been able to "see" Newtonian mechanics displayed in real numbers much more frequently than before and in a greater variety of circumstances. The ability to see data that shows a maximum acceleration for a mass on a spring, while the mass is actually at rest, goes a long way toward developing a Newtonian perspective.

- The increased data acquisition capability and graphical analysis have revealed that our students have a surprising amount of difficulty in visualizing the graphical interpretation of algebraic functions. Perhaps the speed and simplicity that computers bring to the graphical procedure has come at the price of reduced qualitative understanding of what a graph should "look like". Just as the "back of envelope" calculation means more when you previously did it the ten page way, so perhaps more time should be spent in manual graphing to develop a qualitative sense of what constitutes a reasonable graph for an algebraic expression. To address this issue we have required that all lab reports contain at least one hand drawn graph which is used in performing the final data analysis. For example, in the study of a mass attached to a spring students will use the Sonic Ranger to obtain the period of the vibrating mass, but they must then use a hand drawn graph of mass vs. the square of the period to determine the spring constant.

- Many students are reluctant to accept the lack of explicit directions for performing the experiments. They would prefer an explicit experimental design, a pre-arranged data table, and directions for analysis, i.e., they feel more at home with the "cookbook". Since we have used unstructured laboratories with success in the algebra based physics lab (populated by junior and senior level biology students) this unease with lack of structure might simply reflect freshman anxiety. Whatever the source, the student frustration is there and needs to be frequently addressed.

- The transfer of LabVIEW into the upper division laboratories in physics, electrical engineering, and chemistry has been quite successful. There is significant opportunity here for interdisciplinary interaction among both students and faculty. The common exposure to the LabVIEW environment at the freshman year provides an effective foundation for later collaboration.

Finally it is worth reporting some management aspects of introducing this kind of laboratory environment. 
- The lab does not run itself. The maintenance of the network and the computer systems requires time, often lots of it.

- Although the initial learning curve for LabVIEW may appear steep, the reward in rapid development of new experiments is well worth the effort (All of the VI's described above are available by contacting the author.). For example, using the modules we have described, it would be a rather easy task to develop a VI that would simultaneously access a Sonic Ranger and a force sensor. With this VI one could then use repelling magnets, one on an air cart and one on the force sensor, and simultaneously measure the repulsion force and magnet separation.

These two data sets could be combined, with an integration of force to get the potential energy function and differentiation to get the air cart velocity, to explore energy conservation in a rather complex system. Creating this type of experiment using off-the-shelf commercial products targeted to the introductory physics lab would be much more difficult.

\section{Acknowledgments}

I would like to thank Robert Chambers at the University of Arizona for introducing me to the "plan/do" approach to the introductory laboratory. I am also indebted to my colleagues in physics Mark Utlaut, Tom McGlinn, and the University of Portland computer support staff for their help (past and present) in this project. Finally I would like to thank the adjunct lab instructors: Ellain LaJoie, Bruce Meeves, and Greg Mulder, for their often heroic effort.

\section{References}

1. David Hestenes (1987), "A Modeling theory of physics instruction", Am J. Phys, 55, 440-454.

2. David Hestenes (1992), "Modeling games in a Newtonian World", Am J. Phys, 60, 732-748.

3. R. R. Borchers (1988), "In Praise of Visualization and Simulation ", Computers in Physics Jan/Feb )

4. P.W. Laws (1988) in The Conference on Computers in Physics Instruction, E. F. Redish and J. S. Risley, Eds, Addison Wesley.

5. P. W. Laws, et al.(1989), Workshop Physics Activity Guide, 1989-90.

6. L.C. McDermott (1991), "Millikan Lecture (1990): What we teach and what is learned-Closing the gap", Am J. Phys, 59, 301-315.

7. L.C. McDermott and P.S. Shaffer (1992), "Research as a guide for curriculum development: An example from introductory electricity. Part I: Investigation of student understanding, Am J. Phys, 60, 994-1003.

8. D. Rappaport (1989),"Visualizing Physics", Computers in Physics Sept/Oct., p.19.

9. D.R.Sokoloff and R.K. Thornton (1990), Tools for Scientific Thinking, Motion and Force, Vernier Software.

10. R.K. Thornton and D.R.Sokoloff (1990), "Learning motion concepts using real time microcomputer-based laboratory tools", Am.J. Phys, 58, 452-462.

11. Pasco Scientific, 1996 Physics Catalog. 
12. Pricilla Laws, Workshop Physics Activity Guide, Modules 1-4, Wiley, (1997).

13. Central Scientific Company, 1996 Physics Apparatus Catalog.

14. NSF-ILI grant \# DUE-9350747

15. Edward I. Shibata and Daniel MacIsaac, Physics 152 Laboratory Manual, 3'rd Ed., Kendall/Hunt, Dubuque, IA.

RICHARD A. YOUNG received a B.S. in Engineering Physics from Lehigh University and a Ph.D. in theoretical condensed matter physics from the University of Chicago. Prior to coming to the University of Portland he was a member of the physics faculty at the University of Arizona, and a Member of Technical Staff at Bellcore. His research interests include path integral methods and quantum mechanical tunneling phenomena.

\section{cäëE=K-ãE=W=doNKomp=}

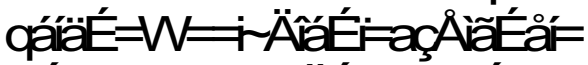

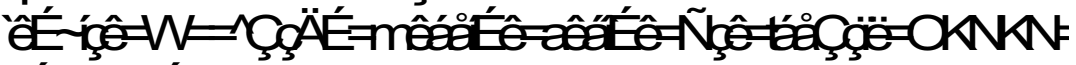

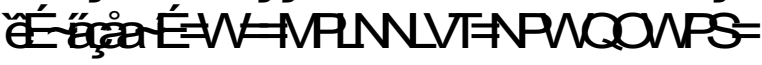

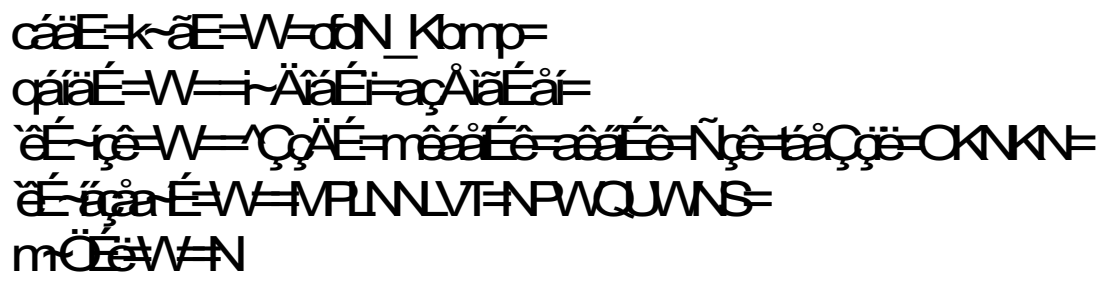

Figure 1. Illustration of the LabVIEW Front Panel and Block Diagram for the determination of the friction torque on a rotating pulley as it slows down. 


\section{cäëE=k-ãE=W=adOKbmp

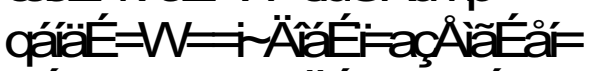

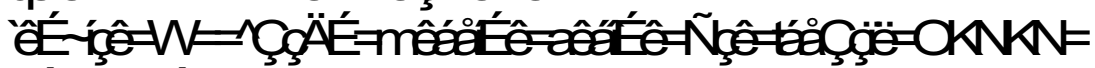

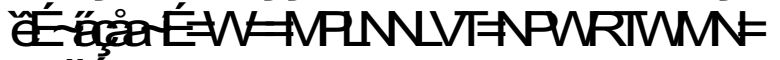 \\ mË}

Figure 2. Illustration of the $(\mathrm{x}, \mathrm{v}, \mathrm{a})$ data analyzer showing the angular displacement, velocity, and acceleration of the pulley over a two second interval. The buttons in the lower right corner terminate this VI and pass the data selected to a second data analysis VI.

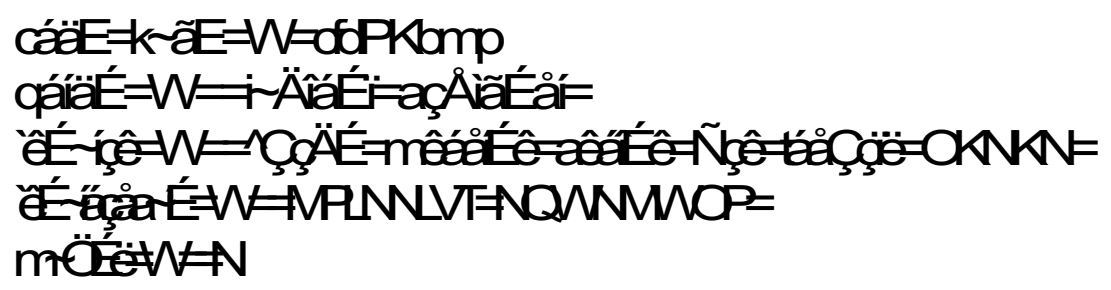


Figure 3. Illustration of the angular velocity data when displayed by the quantitative data analyzer VI. The data is fit to a straight line and indicates that the friction torque is nearly constant as the pulley slows.

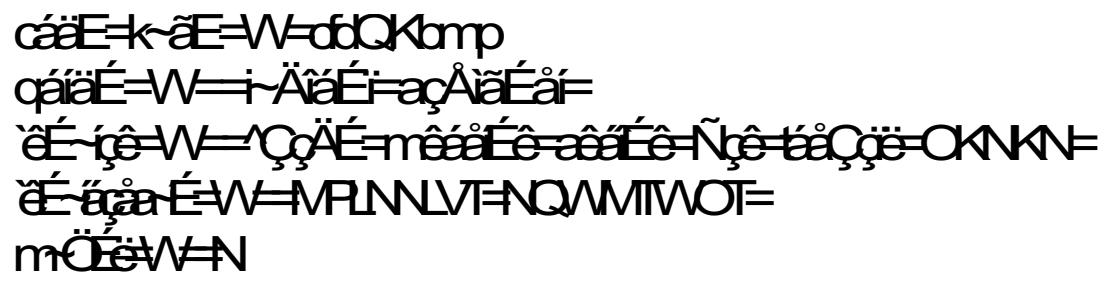

Figure 4. Illustration of the same data as Figure (3) but plotted on a semi-log scale to test a model that the friction torque is proportional to the angular velocity. The larger deviation from the straight line fit indicates that this model is not as good as the constant torque model. 